\title{
Going beyond the final impression with radiologic data in the era of clerical medicine: a case report of coronary artery disease found incidentally on computed tomography
}

\author{
Andrea M. Lauffer, MD
}

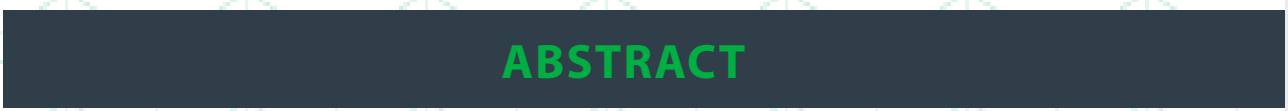

INTRODUCTION: Given the challenges in modern healthcare, physicians find themselves devoting a great deal of mental and emotional energy into executing the appropriate care for patients as well as performing their daily clerical duties. Many clinicians have resorted to innovating ways to minimize their tasks which often leads to narrowing the focus of a patient's overall care and the birth of medical errors described in the Swiss cheese effect model.

CASE Description: A 66 year old male established care with a new primary care physician. Previous radiologic data revealed a pulmonary nodule that did not have appropriate follow up imaging. Repeat imaging showed a stable nodule in the final impression of the report. In the details of the report, coronary artery disease was noted, but it was not mentioned in the conclusion of the report. Patient was referred to cardiology for risk stratification and assessment, and he was found to have multi-vessel coronary artery disease requiring immediate coronary artery bypass grafting.

CONCLUSION: There is a demand for physicians to be efficient and productive in the face of an ever growing difficult healthcare culture. This culture creates a medium in which approach to patient care could be dangerously narrowed. This case illustrates that a final impression of a radiologic report should not be the only item read. A physician's standard approach should be to look at the report as a whole to appropriately treat diagnosed and previously undiagnosed pathologies in order to decrease patient morbidity and mortality.

\section{KEYWORDS}

electronic health record, coronary artery disease, communication, search for satisfaction, radiology, primary care, Swiss cheese effect

\section{INTRODUCTION}

Our current healthcare culture has clinicians battling many health epidemics on a daily basis while facing the pressures of maintaining productivity and efficiency in their practices. Physicians are caring for our general population at the peak of one of the most unhealthy times in history. With ongoing obesity and opioid epidemics and a growing senior citizen population, physicians find themselves devoting a great deal of mental and emotional energy into executing the appropriate care for patients. At the
Author affiliations are listed at the end of this article.

Correspondence to: Andrea Lauffer, MD St. Mary's Medical Center lauffer1@marshall.edu same time, given the skyrocketing costs of healthcare, clinicians find themselves often dealing with insurance companies and engaging in peer to peer conferences in order to get financial approval for the care needed for their patients. The draining costs of healthcare are taking a monetary toll on healthcare facilities that employ many doctors and midlevel providers. Physicians feel pressured to maintain a level of productivity with regard to the volume of patients they see. The balance of productive and efficiency in this given environment is a challenging scenario, especially with the birth of the electronic 
health record (EHR). As a result, a major concern is the advent of physician burnout which can ultimately lead to decreased quality of care for patients.

Given the above obstacles, many clinicians have resorted to innovating ways to minimize their tasks which often leads to narrowing the focus of a patient's overall care. When the focus becomes too narrow, the defenses in preventing medical errors are crippled. This case highlights the need for physicians to be broad in their approaches to patient care despite the high burden of distractions and outside pressures that plague our healthcare system. This case is a reminder that a physician's first and most important obligation is to the patient and to the oath to do no harm.

\section{CASE DESCRIPTION}

A 66 year old Caucasian male presents to a new primary care physician to establish care in May 2017. He had been under the care of another physician whose practice had dissolved The patient was in need of a new primary care doctor. A thorough history and physical was done at the initial visit by the clinician. Pertinent past medical history included obesity, hypertension, hyperlipidemia, and type two diabetes mellitus. Patient's social history revealed a history of tobacco use. With regard to family history, his father had coronary artery disease.

Previous radiologic data was reviewed, and a pulmonary nodule was noted on an abdominal computed tomography scan from 2010. Follow up imaging was recommended at the time, however, the patient never had this done. Given his history of tobacco use, his new primary care physician ordered a repeat computed tomography scan of the chest to assess the stability of the nodule.

Patient underwent computed tomography of the chest without contrast in June 2017. Final impression of the radiologic report was as follows: "Stable benign $7 \mathrm{~mm}$ left lower lobe nodule. A $5 \mathrm{~mm}$ lingular nodule was not included on the prior study. Would consider follow-up exam in 6-12 months."

Further review of the radiologic report showed a diagnosis of coronary artery disease was listed in the interpretive details of the report, but it was not included in the final impression. Patient did not have a previous history of coronary artery disease.

He was subsequently referred to cardiology for further evaluation and risk stratification in July 2017. At his visit with the cardiologist, an EKG was done showing sinus rhythm and no ST changes. The cardiologist reviewed the computed tomography images of the chest and noted fairly extensive calcification at the proximal left anterior descending artery level toward the left main artery. Cardiologist ascertained that while the patient maintained an active life, he did note shortness of breath with exertion. He denied chest pressure or chest pain. Given the patient's symptom of exertional dyspnea, his abnormal CT findings, and his other risk factors significant for coronary artery disease, the cardiologist decided to proceed with a treadmill Cardiolite stress test to assess for ischemia.

Patient had a positive treadmill Cardiolite stress test. As a result, he underwent further assessment of his coronary arteries via a left heart catheterization. He was found to have extensive multi-vessel coronary artery disease not amenable to stenting. He was also noted to have reduced left ventricular function with an ejection fraction of $40 \%$. He was immediately admitted, and the cardiovascular-thoracic surgeon was consulted. He underwent coronary artery bypass grafting successfully.

\section{DISCUSSION}

A publication released by the Ulster Medical Society discusses various causes of missed or untreated diagnoses from radiologic reports. One of the issues highlighted is the "satisfaction of search" phenomenon. 1 While this is a dangerous pitfall for a practicing radiologist, it is equally treacherous for the ordering physician interpreting the report. "Satisfaction of search" phenomenon occurs when an abnormal finding causes one to terminate the search for other abnormalities that could either be relevant or unrelated to the current clinical scenario.' Had this phenomenon occurred in this case, the results could have led to a potentially devastating 
or life ending myocardial infarction for the patient. Fortunately, the ordering clinician in this case reviewed the whole report instead of solely focusing on the assessment of the pulmonary nodule.

Another discussed cause of medical error secondary to radiologic reports is poor communication in which disease is identified and interpreted correctly, but the message fails to reach the relevant clinician. ${ }^{4}$ This certainly could have occurred with this patient since the radiologist did not include the diagnosis of coronary artery disease in the final impression and did not directly communicate with the ordering clinician about the finding of coronary artery disease. However, fortunately the ordering clinician reviewed the entirety of the $C T$ report and did not overlook the presence of coronary artery disease.

One may ask how to safeguard from such potentially life threatening errors. In the Swiss cheese model proposed by James Reason (Image 1), layers of the cheese serve as defenses. ${ }^{1,2}$ Increased layers lead to increase defenses. Holes in our defenses for medical error prevention are continually shifting their locations due to active failures and latent conditions. ${ }^{1,2}$ When the holes in these defenses line up momentarily, opportunities for patient morbidity

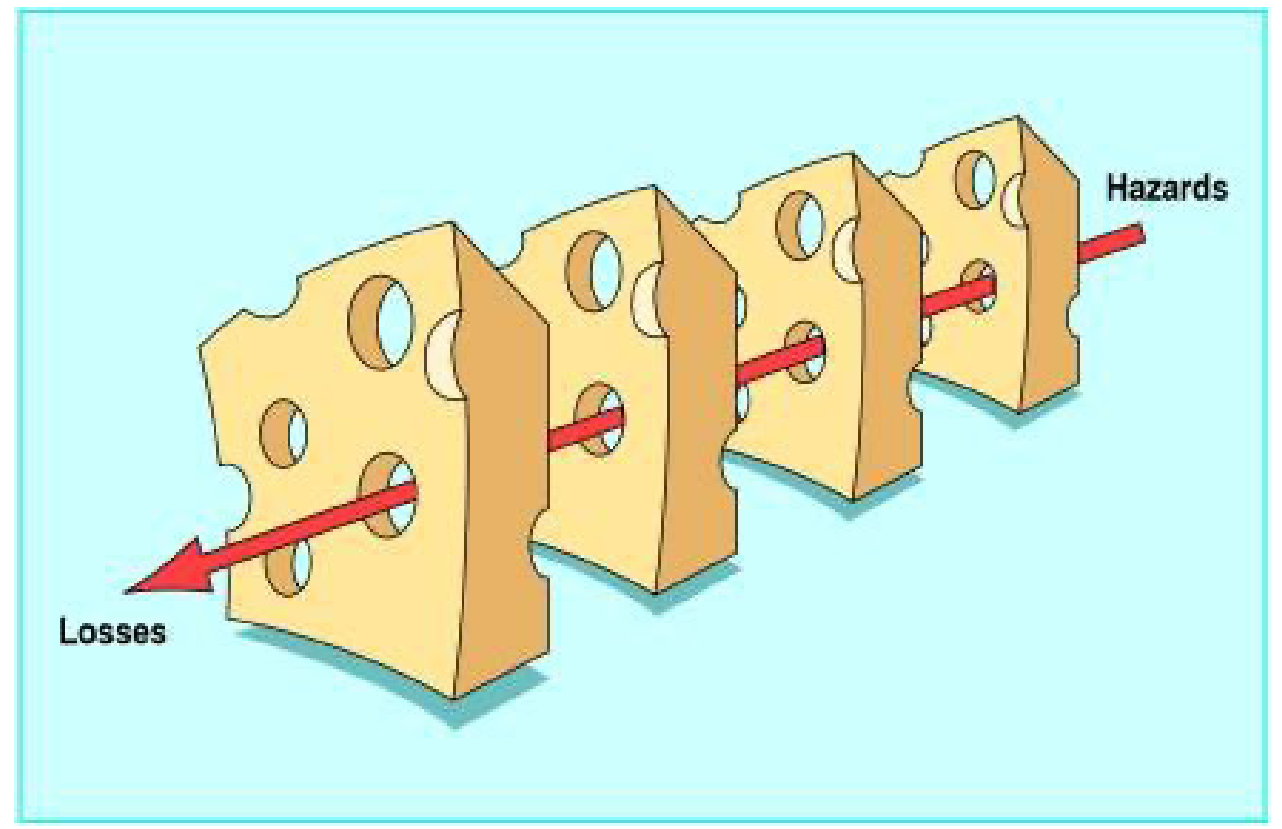

IMAGE 1: Reproduced from Human error: models and management, James Reason, volume number 320, page 769, copyright 2000] with permission from BMJ Publishing Group Ltd. and mortality are born. Active failures are the unsafe acts committed directly to the patient. ' Latent conditions are created by designers of the system that can lead to issues facing physicians today such as time pressure and fatigue. ${ }^{1}$

The birth of the EHR has led to the possible development of a latent condition. According to a study by the Annals of Internal Medicine, for every hour a physician spends with patients, the physician spends two hours on the electronic health record. ${ }^{5}$ In this particular study, sixty physicians from sixteen practices were observed. ${ }^{5} 49 \%$ of the physicians' hours were spent on EHR and deskwork. ${ }^{5}$ Only $27 \%$ of time was spend directly with a patient. ${ }^{5}$

According to a survey by the New England Journal of Medicine Catalyst council which comprised of answers from 570 medical providers including healthcare executives and clinicians, clerical burden is the leading cause of physician burnout. ${ }^{3}$ Clerical burden is largely due to the expanded and more comprehensive use of electronic health records. ${ }^{3}$ EHRs can disrupt the routine workflow already established by physicians. ${ }^{3}$ The physician is often forced to carry clerical duties into after hours. ${ }^{3}$ NEJM Catalyst Council members cite decreased quality of care as the top reason to address the ongoing issue of physician burnout (Image 2). ${ }^{3}$

\section{CONCLUSION}

With the demand for physicians to be efficient and productive in the face of an ever growing difficult healthcare culture, one could argue that the designers of our healthcare system have created the opportunity for latent conditions to manifest, particularly physician burnout. Consequently, 


\section{Increased Clerical Burden and Productivity \\ Requirements/Expectations Produce Physician Burnout}

What are the top two factors contributing to the increase in physician burnout?

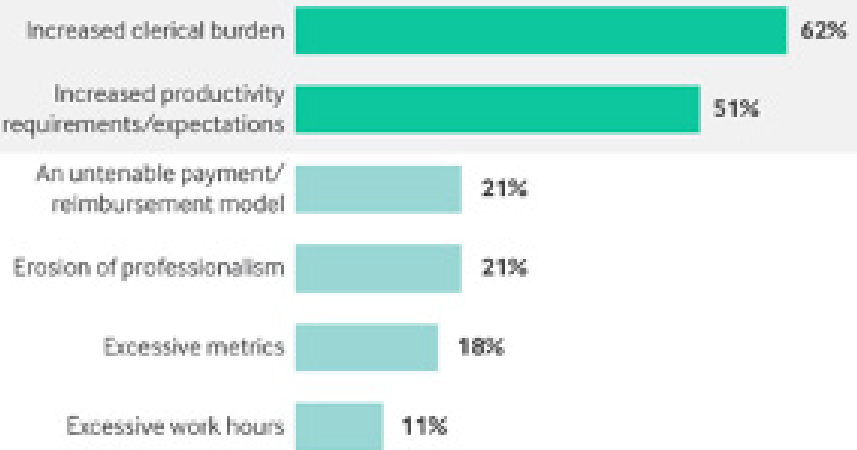

A higher percentage of Executives than Clinical Leaders and Clinicians cite increased clerical burden as a top factor in increased physiclan burnout
$72 \%$

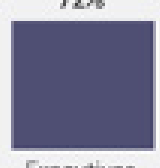

Executives

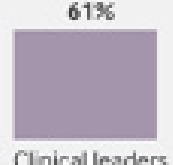

Clnical leaders

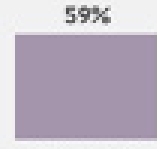

Clinicians
Base $=570$ imuitiple responses:

NGM Cesalyst kasalpst nejm orgl e Messachusetts Medical Society

IMAGE 2: Why Physician Burnout Is Endemic \& How Health Care Must Respond [Internet]. NEJM Catalyst. 2017 [cited 20170ct5]. Available from: https://catalyst.nejm. org/physician-burnout-endemic-healthcare-respond/ Image reposted from NEJM Catalyst.

this allows the chance for a decrease in quality of patient care and the opportunity for the dreaded Swiss cheese effect to occur. Despite the burden of complex patient care and the new age in which a physician needs a great deal of clerical time to complete daily tasks, it is imperative that physicians make conscientious efforts to thoroughly review all patient clinical data. With regard to the relevancy of this case report, this applies especially to radiology reports. Final impression of a radiology report should not be the only item read, and a physician's standard approach should be to look at the report as a whole to appropriately treat diagnosed and previously undiagnosed pathologies.

\section{AUTHOR AFFILIATIONS}

\author{
1. St. Mary's Medical Center, \\ Huntington, West Virginia
}

\section{REFERENCES}

1. Reason J. Human error: models and management [Internet]. BMJ: British Medical Journal; 2000 [cited 20170ct4]. Available from: https://www.ncbi.nlm. nih.gov/pmc/articles/PMC1117770/

2. Systems [Internet]. CMPA Good Practices Guide - What is "systems thinking". [cited 20170ct5]. Available from: https://www.cmpa-acpm.ca/ serve/docs/ela/goodpracticesguide/ pages/patient safety/Systems/systems thinking 2-e.html

3. Why physician burnout is endemic \& how health care must respond [Internet]. NEJM Catalyst. 2017 [cited 20170ct5]. Available from: https:// catalyst.nejm.org/physician-burnoutendemic-healthcare-respond/

4. Brady A, Laoide RÓ, McCarthy P, McDermott R. Discrepancy and error in radiology: concepts, causes and consequences [Internet]. The Ulster Medical Journal. The Ulster Medical Society; 2012 [cited 20170ct5]. Available from: https://www. ncbi.nlm.nih.gov/pmc/articles/PMC3609674/

5. Half of physician time spent on EHRs and paperwork [Internet]. NEJM Journal Watch. Journal Watch; 2016 [cited 20170ct5]. Available from: http://www.jwatch.org/ fw111995/2016/09/06/half-physician-timespent-ehrs-and-paperwork 